\title{
Decentralized control of inland navigation networks with distributaries: application to navigation canals in the north of France
}

\author{
P. Segovia ${ }^{* * *}$, L. Rajaoarisoa*, F. Nejjari**, V. Puig**, E. Duviella*
}

\begin{abstract}
Inland navigation networks are large-scale systems with large dead times, nonlinearities and variable time delays. They are composed of interconnected reaches. The management goal is to maintain the water level of each reach around a certain value. In this paper, a decentralized control approach is addressed to fulfill this objective. It is based on IDZ models by considering connected reaches with distributaries. A real navigation sub-network in the north of France serves as the case study for this work.
\end{abstract}

\section{INTRODUCTION}

Inland navigation networks cover more than 37,000 kilometers in Europe. It is one of the longest networks of inland waterways in the world, offering an interesting alternative to road and rail transport modes [9], [11]. The accommodation of navigation requires an efficient management of the water resource and the control of the water level in each location of the network. Hence, efficient control algorithms of inland navigation networks have to be designed.

Since several years, contributions on automatic control of water systems are extensively proposed in literature. Survey of these contributions are proposed in [7] and [10]. Most of them are dedicated to irrigation canals [8], [12], [13], [16], [17] and [4]. More recently, control algorithms have been designed considering characteristics of inland navigation reaches such as resonance, no significant slope or locks in [3] and [5]. They aim at keeping the level close to an operating point by rejecting disturbances due to lock operations. Some specific works deal only with single navigation reach. In [15], a decentralized control algorithm is designed to control the water level of interconnected navigation reaches. A distributed Model-based Predictive Control is also proposed in [1] and [14] to overcome the computational effort of centralized control techniques. In these approaches, systems are simple-shaped reaches without distributary shapes. To the best knowledge of the authors, there is no contribution on decentralized control for openchannel systems with distributaries. Hence, this paper aims at designing a decentralized control algorithm dedicated to interconnected navigation reaches with distributaries. It is based on a modeling step of open water systems that leads to Integral-Delay-Zero models [6]. Then, the decentralized control algorithm is designed guaranteeing stability criterion. Section II of this paper is dedicated to the description of waterway networks characteristics and the management objectives. The modeling approach is detailed in Section III.

* Mines-Douai IA, France. ${ }^{* *}$ Automatic Control Department, Technical University of Catalonia (UPC), Spain. Corresponding author: pablo.segovia@mines-douai.fr
The decentralized control architecture is given in Section IV. Finally, the simulation results are given in Section V, considering a navigation sub-network located in the north of France and highlighting the performance of the designed controller.

\section{PROBLEM DESCRIPTION AND FORMULATION}

\section{A. Management objectives}

Inland navigation networks are large-scale open-flow systems that can be decomposed into several reaches or pools equipped with gates and locks. The main management objective of each reach is to maintain its water level close to the Normal Navigation Level (NNL) as shown in Fig.1, by rejecting disturbances that can be generated by lock operations. Despite being generally not significant, in some cases these disturbances cause the water level to be outside the boundaries defined by LNL (Lower Navigation Level) and HNL (Higher Navigation Level). The dynamics of each

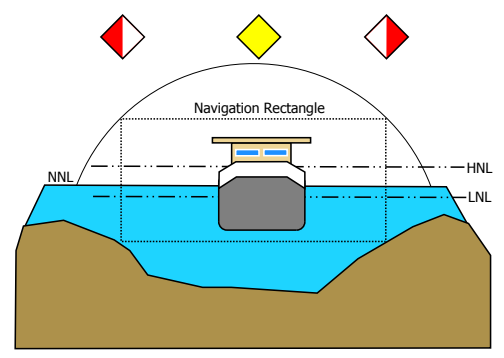

Fig. 1: NNL and navigation rectangle.

reach are nonlinear and exhibit large variable dead times. They are often described by the nonlinear Saint-Venant partial differential equations [2]. It is possible to linearize them by considering a single operating point: the NNL and a close interval around this value (navigation rectangle).

The control problem is quite complex to tackle as the considered system is composed by many reaches (MIMO system), and therefore there exist strong interactions between different pools, with water resources being shared among them. One possible way to fulfill the management objective (NNL) is to consider a decentralized control approach in which each reach is controlled separately. As navigation networks are natural distributed systems, this method is suitable to achieve the control purpose taking into account interactions among the reaches. 


\section{B. Problem formulation}

Consider that the global interconnected system can be described by the following equation:

$$
\Sigma:\left\{\begin{array}{l}
Y(t)=f(U, t), \\
Y\left(t_{0}\right)=Y_{0} \text { with } Y \in \mathbb{R}^{n}, U \in \mathbb{R}^{m}, \text { and } t \in \mathbb{N}
\end{array}\right.
$$

where $t$ defines the continuous time, $U$ the set of the $m$ input terms of the system like gate discharges, $Y$ the $n$ outputs i.e. upstream and downstream water depths, with $Y_{0}$ the water depths at initial time $t_{0} . f$ is a non-linear function. By linearizing the system around around the NNL and using Laplace transform, (1) becomes:

$$
\Sigma: Y(s)=P(s) U(s)
$$

where $s$ is the Laplace variable and $P(s)$ the transfer matrix defined by (3). The structure of the elements of $P(s)$ is given in the next section.

$$
[P(s)]=\left[\begin{array}{cccc}
p_{1,1}(s) & p_{1,2}(s) & \cdots & p_{1, m}(s) \\
p_{2,1}(s) & p_{2,2}(s) & \cdots & \vdots \\
\vdots & \vdots & \ddots & \vdots \\
p_{n, 1}(s) & p_{n, 2}(s) & \cdots & p_{n, m}(s)
\end{array}\right] .
$$

Then, the feedback system $\Sigma_{c l}$ consists of:

$$
\Sigma_{c l}:\left\{\begin{aligned}
\text { Plant } \Sigma: Y(s) & =P(s) U(s), \\
\text { Controller } \Lambda: U(s) & =K(s) e(s), \\
\text { Feedback } \Pi: e(s) & =Y^{d}(s)-Y(s) .
\end{aligned}\right.
$$

where matrices $P(s)$ and $K(s) \in \mathscr{R}(s)$ have dimensions $n \times m$ and $m \times n$, respectively, and belong to the set of real rational matrices, and $Y^{d}$ is the set of the objectives.

While assuming that the feedback system $\Sigma_{c l}$ is well posed, that $\operatorname{det}\left[I_{m}+P(s) K(s)\right] \neq 0$, denote $\Sigma_{i}$ as the subset of $\mathscr{R}(s)$ consisting of proper rational functions whose poles are all in $C_{i} . \Sigma$ is stable if $H(P, K)(s)$ is included in system matrices $\Sigma_{i}$, that is, $C$ stabilizes $\Sigma$.

The global interconnected system can be decomposed as

$$
\Sigma_{c l}:\left\{\begin{aligned}
\Sigma_{i}: y_{i}(s) & =p_{i i}(s) u_{i}(s)+\sum_{j=1}^{m} p_{j i}(s) u_{j}(s) \\
\Lambda: u_{i}(s) & =K_{i i}(s) e_{i}(s)+\sum_{j=1}^{m} K_{j i}(s) e_{j}(s) \\
\Pi: e_{i}(s) & =y_{i}^{d}(s)-y_{i}(s) \\
& \quad \text { for } i=1,2, \ldots, n \text { and } j \neq i
\end{aligned}\right.
$$

where $p_{i i}(s)$ and $K_{i j}(s)$ are sub-elements of $P(s)$ and $K(s)$ respectively. The $i^{t h}$ term $\sum_{j=1}^{m}\left(p_{j i}(s) u_{j}(s)\right)$ is the effect of all inputs on the measurement point $i$. It represents the weighting interconnection values used to model the strength of interconnections. Hence, the control objective is to design a set of optimal output feedback controller defined by:

$$
u_{i}(s)=K_{i i}(s) e_{i}(s)
$$

such that controller $K$ exists and its diagonal blocks are $m$ separate controllers defined by:

$$
K=\operatorname{diag}\left(K_{i i}\right), \quad \forall i=1, \ldots, m
$$

and shows in the same way that the local feedback action is sufficient to stabilize the subsystem to each other according to its desired performance. Thus, if the interconnection effects of the other subsystems are minimized, the control problem is reduced to the following matrix relationship:

$$
\left[\begin{array}{c}
u_{1} \\
u_{2} \\
\vdots \\
u_{m}
\end{array}\right]=\left[\begin{array}{cccc}
K_{11} & 0 & \cdots & 0 \\
0 & K_{22} & \cdots & 0 \\
\vdots & \vdots & \ddots & 0 \\
0 & 0 & \cdots & K_{m m}
\end{array}\right]\left[\begin{array}{c}
e_{1} \\
e_{2} \\
\vdots \\
e_{m}
\end{array}\right]
$$

To guarantee the convergence of the controller, the following results can be stated [15].

Theorem 1: The decoupled subsystem (5) is locally stabilizable (resp. stable) by a proportional output feedback control law

$$
u_{i}(s)=K_{i i} e_{i}(s)
$$

such that controller $K$ exists and its diagonal blocks are $m$ separate controllers defined by

$$
K=\operatorname{diag}\left(K_{i i}\right), \quad \forall i=1, \ldots, m
$$

if there exists a symmetric definite positive matrix $\Gamma=\Gamma^{T}>$ 0 where

$$
\mathfrak{L}(\Gamma)={\tilde{A_{i i}}}^{T} \Gamma \tilde{A_{i i}}-\Gamma<0
$$

for any initial output function $y_{i}\left(t_{0}\right)$ with $\tilde{A_{i i}}=\left(A_{i i}-B_{i i} K_{i i}\right)$, with $A_{i i}$ and $B_{i i}$ elements of state space matrices $A$ and $B$.

Theorem 2: The coupled subsystem (5) is globally stabilizable (resp. stable) by a proportional output feedback control law

$$
u_{i}(s)=\left(K_{i, i} e_{i}(s)+\sum_{j=1}^{m} \Gamma_{i, j}\right) \text { with } j \neq i \text { and for } i=1, \ldots, n
$$

if

- the system is locally stable,

- there exist symmetric definite positive matrices $Q=$ $Q^{T}>0$ and $\Gamma=\Gamma^{T}>0$ which satisfy the following conditions

$$
\begin{gathered}
C r_{1}=\tilde{A_{i i}}{ }^{T} \underline{Q A_{i i}}-Q<0 \\
C r_{2}={\tilde{A_{i j}^{T}}}^{n} \mathfrak{L}(\Gamma) \tilde{A_{i j}}{ }^{n}<0 \text { with } n \in \mathbb{N}
\end{gathered}
$$

for any initial state function $x\left(t_{0}\right)$.

Proof: Proof is omitted here due to the lack of space. The reader is referred to [15] and [20] to prove these two theorems.

\section{MODELING OF INLAND NAVIGATION NETWORKS}

The modeling approach is presented in a generic way, so that its application can be extended to as many particular cases as possible. A canal is usually modeled by dividing it into reaches, which are portions of a canal comprised between two hydraulic structures. However, canals may be divided into pools even if there is no such hydraulic structure. For instance, a sudden geometrical change in the canal can lead to considering two different reaches. The first step is to consider single pools and obtain their models individually. Once this goal is achieved, the complete network can be 
built by interconnecting the individual pools. Many different configurations may exist in a network, depending on how these reaches are connected.

\section{A. Identifying the parameters of a single reach}

The Integrator Delay Zero (IDZ) model is proposed in [6] as an enhancement of the Integrator Delay (ID) model. The structure of the IDZ model is as follows:

$$
p_{i j}(s)=\frac{\alpha s+1}{\mathscr{A} s} e^{-\tau s},
$$

where $\alpha$ represents the inverse of the transfer function's zero, $\mathscr{A}$ the integrator gain and $\tau$ the propagation time delay (which is equal to 0 when $i=j$ since the origin and the measurement point of the action are the same). The exact values of these parameters cannot be computed, but an accurate approximation is provided in [6] nevertheless. This fact, as it can later be seen in Section III-B, causes the change in the notation of these functions from $p_{i j}(s)$ to $\hat{p}_{i j}(s)$.

In low frequencies, the canal behaves like a tank, and the integrator term captures this phenomenon. It can be approximated by the reciprocal of the backwater area (surface of the canal reach). The delay represents the minimum time required for a wave to travel from its point of origin to the point of measurement. For constant wave velocity $V$ and wave celerity $C_{w}$, two different time delays ( $\tau_{d}$ the time from upstream to downstream, $\tau_{u}$ from downstream to upstream) are computed:

$$
\begin{aligned}
\tau_{u} & =\frac{L}{C_{w}-V} \\
\tau_{d} & =\frac{L}{C_{w}+V}
\end{aligned}
$$

where $L(\mathrm{~m})$ is the total length of the canal, $C_{w}=\sqrt{g \mathscr{A} / T}$ and $V=q / \mathscr{A}(\mathrm{m} / \mathrm{s})$, with $g\left(\mathrm{~m} / \mathrm{s}^{2}\right)$ the gravitational acceleration, $\mathscr{A}\left(\mathrm{m}^{2}\right)$ the section, $T(\mathrm{~m})$ the top width of the canal section and $q\left(\mathrm{~m}^{3}\right)$ the discharge.

In high frequencies, the dynamics are dominated by the zero of (12). By adding it to the integrator delay terms, one obtains a model that provides good fits in the whole frequency range. To calculate this zero, one must refer to the procedure detailed in [6].

The aforementioned transfer function can be used to model any reach. Considering a reach with two measured depths $y(0, s)$ and $y(L, s)$, and two discharges $q(0, s)$ and $q(L, s)$, at upstream $(x=0)$ and downstream $(x=L)$ points, the expression that relates the discharges to the water depths is denoted by the $2 \times 2$ transfer matrix:

$$
\left[\begin{array}{l}
y(0, s) \\
y(L, s)
\end{array}\right]=\underbrace{\left[\begin{array}{ll}
\hat{p}_{11}(s) & \hat{p}_{12}(s) \\
\hat{p}_{21}(s) & \hat{p}_{22}(s)
\end{array}\right]}_{P(s)}\left[\begin{array}{c}
q(0, s) \\
q(X, s)
\end{array}\right],
$$

with $P(s)$ the transfer function matrix whose elements $\hat{p}_{i j}(s)$ follow the IDZ structure presented in (12). The general procedure on how to obtain the parameters $\alpha, \mathscr{A}$ and $\tau$ for all $\hat{p}_{i j}(s)$ elements (14) can be found in detail in [6].

\section{B. Computing the interconnection of different reaches}

Once it is clear how to obtain the model of a pool, the next goal is to obtain a procedure that makes it possible to interconnect successive pools in order to build the global model of the network. The basic studied structures are depicted in Fig.2.

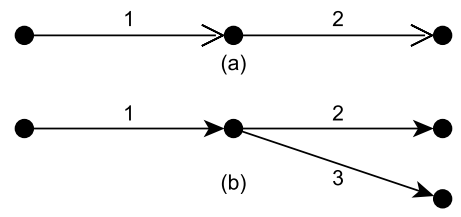

Fig. 2: (a) Simple interconnection. (b) Distributary.

The reason for studying basic structures is that they are the ones that constitute more complex configurations. Particular network configurations can be studied by applying the same steps followed here.

1) Simple interconnection: This situation refers to two consecutive reaches that are linked by a common node in the absence of any hydraulic structure, and each of these parts presents a uniform geometry (see Fig.2a.). In this case, according to [6],

$$
\begin{aligned}
& y_{0}^{(\kappa)}=\hat{p}_{11}^{(\kappa)}(s) q_{0}^{(\kappa)}+\hat{p}_{12}^{(\kappa)}(s) q_{X}^{(\kappa)} \\
& y_{X}^{(\kappa)}=\hat{p}_{21}^{(\kappa)}(s) q_{0}^{(\kappa)}+\hat{p}_{22}^{(\kappa)}(s) q_{X}^{(\kappa)}
\end{aligned}
$$

with $\hat{p}_{i j}(s)$ representing the estimated model for the theoretical $p_{i j}(s)$ function, and with $\kappa=1$ for the upstream reach and $\kappa=2$ for the downstream reach. The global model is then obtained by imposing the following conditions; $y_{X}^{(1)}=y_{0}^{(2)}$ and $q_{X}^{(1)}=q_{0}^{(2)}$ :

$$
\begin{aligned}
& y_{0}^{(1)}=\hat{p}_{11}^{(G)}(s) q_{0}^{(1)}+\hat{p}_{12}^{(G)}(s) q_{X}^{(2)} \\
& y_{X}^{(2)}=\hat{p}_{21}^{(G)}(s) q_{0}^{(1)}+\hat{p}_{22}^{(G)}(s) q_{X}^{(2)}
\end{aligned}
$$

where $\hat{p}_{i j}^{(G)}$ represent the interconnected IDZ transfer functions. Their expressions are:

$$
\begin{gathered}
\hat{p}_{11}^{(G)}=\hat{p}_{11}^{(1)}+\frac{\hat{p}_{12}^{(1)} \hat{p}_{21}^{(1)}}{\hat{p}_{11}^{(2)}-\hat{p}_{22}^{(1)}} \\
\hat{p}_{12}^{(G)}=-\frac{\hat{p}_{12}^{(1)} \hat{p}_{12}^{(2)}}{\hat{p}_{11}^{(2)}-\hat{p}_{22}^{(1)}} \\
\hat{p}_{21}^{(G)}=\frac{\hat{p}_{21}^{(1)} \hat{p}_{21}^{(2)}}{\hat{p}_{11}^{(2)}-\hat{p}_{22}^{(1)}} \\
\hat{p}_{22}^{(G)}=\hat{p}_{22}^{(2)}-\frac{\hat{p}_{12}^{(2)} \hat{p}_{21}^{(2)}}{\hat{p}_{11}^{(2)}-\hat{p}_{22}^{(1)}}
\end{gathered}
$$

where the Laplace variable $s$ is omitted for readability in all $\hat{p}_{i j}^{(\kappa)}$ terms.

2) Distributary: Also known in hydrology as effluent, it can be defined as a stream that branches off from the main stream and flows away. The model for each reach is described by (14) and the conditions for this particular case are: 


$$
\begin{aligned}
& y_{X}^{(1)}=y_{0}^{(2)}=y_{0}^{(3)} \\
& q_{0}^{(2)}=\lambda q_{X}^{(1)} \\
& q_{0}^{(3)}=(1-\lambda) q_{X}^{(1)}
\end{aligned}
$$

where $\lambda \in(0,1)$ indicates that the flow is divided between the two streams after the node.

The interconnected model for the distributary case is:

$$
\begin{aligned}
& y_{0}^{(1)}=\hat{p}_{11}^{(G)}(s) q_{0}^{(1)}+\hat{p}_{12}^{(G)}(s) q_{X}^{(2)}+\hat{p}_{13}^{(G)}(s) q_{X}^{(3)} \\
& y_{X}^{(2)}=\hat{p}_{21}^{(G)}(s) q_{0}^{(1)}+\hat{p}_{22}^{(G)}(s) q_{X}^{(2)}+\hat{p}_{23}^{(G)}(s) q_{X}^{(3)} \\
& y_{X}^{(3)}=\hat{p}_{31}^{(G)}(s) q_{0}^{(1)}+\hat{p}_{32}^{(G)}(s) q_{X}^{(2)}+\hat{p}_{33}^{(G)}(s) q_{X}^{(3)}
\end{aligned}
$$

with

$$
\begin{aligned}
& \hat{p}_{11}^{(G)}=\hat{p}_{11}^{(1)}+\hat{p}_{12}^{(1)} \hat{p}_{21}^{(1)}\left[\frac{\lambda}{\lambda \hat{p}_{11}^{(2)}-\hat{p}_{22}^{(1)}}+\frac{1-\lambda}{(1-\lambda) \hat{p}_{11}^{(3)}-\hat{p}_{22}^{(1)}}\right] \\
& \hat{p}_{12}^{(G)}=-\frac{\lambda \hat{p}_{12}^{(1)} \hat{p}_{12}^{(2)}}{\lambda \hat{p}_{11}^{(2)}-\hat{p}_{22}^{(1)}} \\
& \hat{p}_{13}^{(G)}=-\frac{(1-\lambda) \hat{p}_{12}^{(1)} \hat{p}_{12}^{(3)}}{(1-\lambda) \hat{p}_{11}^{(3)}-\hat{p}_{22}^{(1)}} \\
& \hat{p}_{21}^{(G)}=\lambda \hat{p}_{21}^{(1)} \hat{p}_{21}^{(2)}\left[\frac{\lambda}{\lambda \hat{p}_{11}^{(2)}-\hat{p}_{22}^{(1)}}+\frac{1-\lambda}{(1-\lambda) \hat{p}_{11}^{(3)}-\hat{p}_{22}^{(1)}}\right] \\
& \hat{p}_{22}^{(G)}=\hat{p}_{22}^{(2)}-\frac{\lambda^{2} \hat{p}_{12}^{(2)} \hat{p}_{21}^{(2)}}{\lambda \hat{p}_{11}^{(2)}-\hat{p}_{22}^{(1)}} \\
& \hat{p}_{23}^{(G)}=-\frac{\lambda(1-\lambda) \hat{p}_{21}^{(2)} \hat{p}_{12}^{(3)}}{(1-\lambda) \hat{p}_{11}^{(3)}-\hat{p}_{22}^{(1)}} \\
& \hat{p}_{31}^{(G)}=(1-\lambda) \hat{p}_{21}^{(1)} \hat{p}_{21}^{(3)}\left[\frac{\lambda}{\lambda \hat{p}_{11}^{(2)}-\hat{p}_{22}^{(1)}}+\frac{1-\lambda}{(1-\lambda) \hat{p}_{11}^{(3)}-\hat{p}_{22}^{(1)}}\right] \\
& \hat{p}_{32}^{(G)}=-\frac{\lambda(1-\lambda) \hat{p}_{12}^{(2)} \hat{p}_{21}^{(3)}}{\lambda \hat{p}_{11}^{(2)}-\hat{p}_{22}^{(1)}} \\
& \hat{p}_{33}^{(G)}=\hat{p}_{22}^{(3)}-\frac{(1-\lambda)^{2} \hat{p}_{12}^{(3)} \hat{p}_{21}^{(3)}}{(1-\lambda) \hat{p}_{11}^{(3)}-\hat{p}_{22}^{(1)}}
\end{aligned}
$$

where the Laplace variable $s$ is again omitted for readability.

\section{CONTROL DESIGN}

The control objective is to keep the levels close to the setpoint by controlling the discharges without violating the physical constraints of the system. The discharges have to satisfy a lower $\left(l_{b}\right)$ and upper $\left(u_{b}\right)$ bound constraints as:

$$
l_{b} \leq u(t) \leq u_{b}
$$

The continuous-time transfer functions from (4) is discretized and a minimal realization in state-space representation of the system is computed. While using standard tools, the minimal state-space representation of the system (5) by using the IDZ equation (12) is:

$$
\Sigma_{s s}:\left\{\begin{aligned}
x(k+1)= & A_{0} x(k)+A_{1} x(k-\tau) \\
& +B_{0} u(k)+B_{1} u(k-\tau) \quad x\left(k_{0}\right)=x_{0} \\
y(k)= & C x(k)+D u(k),
\end{aligned}\right.
$$

where $k \in \mathbb{N}$ defining the discrete time. $x \in \mathbb{R}^{r}, u \in \mathbb{R}^{m}, y \in$ $\mathbb{R}^{n}, A_{0}$ and $A_{1} \in \mathbb{R}^{r \times r}, B_{0}$ and $B_{1} \in \mathbb{R}^{r \times m}, C \in \mathbb{R}^{n \times r}, D \in$ $\mathbb{R}^{n \times m}$, composed of $\beta$ linear time-invariant of subsystems $\Sigma_{i}$, described by:

$$
\Sigma_{s s_{i}}:\left\{\begin{aligned}
x_{i}(k+1)= & A_{i i} x_{i}(k)+B_{i i} u_{i}(k)+\sum_{j=1}^{\beta} \\
& {\left[A_{i j} x_{j}\left(k-\tau_{i j}\right)+B_{i j} u_{j}\left(k-\tau_{i j}\right)\right] } \\
& \text { for } i=1,2, \ldots, \beta \text { and } j \neq i \\
y_{i}(k)= & C_{i i} x_{i}(k)+D_{i i} u_{i}(k),
\end{aligned}\right.
$$

The considered control inputs are the discharge changes around the operating points. Consequently, the discretized transfer functions must contain an integrator. The reason for this change of inputs is that, due to physical restrictions of the locks involved, the rate of change of the discharge has to be limited. By using the change of the discharge and not the discharge, the bounds (21) are incorporated into the formulation of the controller design problem.

The control design problem is described in this case by:

$$
u_{i}(k)=K_{i i} e_{i}(k),
$$

where the decentralized optimal proportional controller $K$ is computed according to the minimization of the quadratic cost function [5] :

$$
\begin{aligned}
\min _{u_{i}} J= & \sum_{l=1}^{\delta}\left(e_{i}(k+l \mid k)^{T} S e_{i}(k+l \mid k)\right. \\
& \left.+\sum_{l=0}^{\delta-1} u_{i}(k+l \mid k)^{T} R u_{i}(k+l \mid k)\right),
\end{aligned}
$$

subject to the following constraints:

$$
\begin{array}{r}
\forall l=1, \ldots ., \delta: \\
z(k+l+1 \mid k)=\hat{A} z(k+l \mid k)+\hat{B} u(k+l \mid k) \\
e(k+l \mid k)=\hat{C} z(k+l \mid k) \\
l_{b} \leq z_{i}(k+l \mid k) \leq u_{b} \\
z_{i}(k+l \mid k) \in\left[\operatorname{zmin}_{i}, \text { zmax }_{i}\right], \quad \forall i=1, \ldots, m
\end{array}
$$

Here $z_{i}(k+l \mid k)$ denotes the $\mathrm{i}^{\text {th }}$ entry of $z(k+l \mid k)$. In other terms, the resulting state feedback is defined as $g(z)=$ $u^{*}(k \mid k)$ where $u^{*}=(u(k \mid k), u(k+1 \mid k), \cdots, u(k+\delta-1 \mid k))$. In (25), $S$ and $R$ are weighting matrices, or design parameters, where the state-cost matrix $S$ weights the state and the performance index matrix $R$ weights the control effort. If $S$ is increased while $R$ remains constant, the settling time will be reduced as the states approach zero at a faster rate. The controller in this case maintains the error sufficiently small to guarantee the convergence of the controller. $\delta$ will serve as prediction horizon to improve the performance of the decentralized controller.

The minimization of (25) can be carried out, for example, by using quadratic programming [18]. In this work, the builtin MATLAB function is used for this purpose [19].

\section{APPLICATION ON INLAND NAVIGATION NETWORK IN THE NORTH OF FRANCE}

The previously described modeling and control techniques are applied on a part of the inland navigation network in the north of France, which is schematized in Fig.3. 


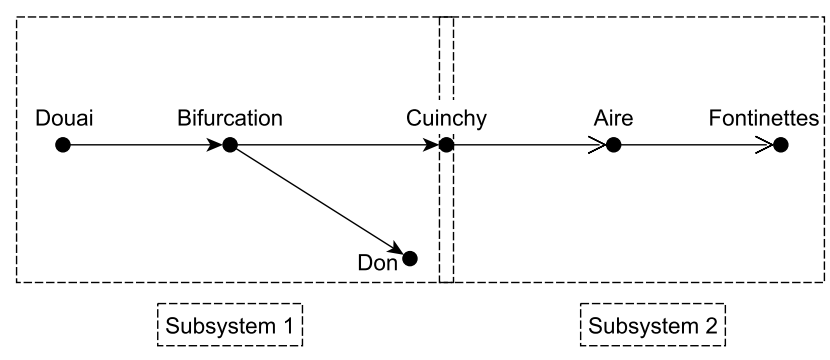

Fig. 3: Case study.

Two adjoining subsystems are considered in this work: first of all, the Douai-Don-Cuinchy reach (DDCR) is bounded at the upstream end by the lock of Douai and at the downstream end by the lock and gate of Cuinchy. Besides, an effluent branches off from the main stream at the bifurcation point and it is bounded downstream by the lock of Don, where there is also a controlled hydraulic structure. So, the main objective is to guarantee the maintain of the water level in each sub-reach between a desired value, by using a local law control but not a global centralized control.

A second reach is considered after the lock and gate of Cuinchy, the Cuinchy-Fontinettes reach (CFR), which is bounded downstream by the lock of Fontinettes. Aire is found halfway between Cuinchy and Fontinettes. The CFR is decomposed in two reaches.

\section{A. Description of the subsystems}

Operation conditions on the locks as well as physical and geometrical parameters for each subsystem are presented hereafter.

1) The Douai-Don-Cuinchy subsystem: The seaworthiness of DDCR is guaranteed if the water level is kept in the interval 3.5 (NNL) $\pm 0.15 \mathrm{~m}$. This reach is supplied by the lock of Douai, with volume equal to $4000 \mathrm{~m}^{3}$. There is a flow bifurcation 27,000 $\mathrm{m}$ downstream: a certain amount of water diverts from the main stream and flows into Don, and the remaining part flows into Cuinchy. The system is emptied by the lock of Cuinchy, with a volume equal to $3,700 \mathrm{~m}^{3}$.

2) The Cuinchy-Fontinettes subsystem: The seaworthiness of CFR in ensured by maintaining the water level within 3.8 $(\mathrm{NNL}) \pm 0.15 \mathrm{~m}$. The reach is supplied by the previous reach, the DDCR, through the lock of Cuinchy. On the other hand, the reach is emptied by the lock of Fontinettes, which must overcome a larger volume of $25,000 \mathrm{~m}^{3}$. Therefore, the most important disturbances created in this reach are the ones that come from the operation of the lock of Fontinettes.

\begin{tabular}{ccccccc}
\hline \hline & $n_{r}$ & $m_{s}$ & $B_{w}$ & $\mathrm{q}$ & $\mathrm{L}$ & $y_{X}$ \\
\hline Douai-BIF & 0.035 & 0 & 52 & 1 & 27000 & 3.5 \\
\hline BIF-Don & 0.035 & 0 & 52 & 0.5 & 5200 & 3.5 \\
\hline BIF-CUI & 0.035 & 0 & 52 & 0.5 & 11000 & 3.5 \\
\hline \hline CUI-AI & 0.035 & 0 & 52 & 0.6 & 28700 & 3.8 \\
\hline AI-FON & 0.035 & 0 & 52 & 0.6 & 13600 & 3.8 \\
\hline \hline
\end{tabular}

TABLE I: Physical data for DDCR and CFR.

Table I sums up the physical and geometrical data used to model the DDCR: $n_{r}\left[\mathrm{~s} / \mathrm{m}^{1 / 3}\right]$ is Manning's roughness coefficient, $m_{s}$ (dimensionless) is the side slope of the cross section ( $m=0$ for rectangular shape), $B_{w}[m]$ is the bottom width of the reach, $q\left[\mathrm{~m}^{3} / \mathrm{s}\right]$ is the average flow considering an horizon of one day, $L[\mathrm{~m}]$ is the total length of the pool and $y_{X}[m]$ is the downstream water depth of the reach. Besides, as the canals can be considered flat, the bottom slope $s_{b}$ is equal to 0 for all the reaches. On the other side, BIF, CUI, AI and FON stand for bifurcation, Cuinchy, Aire and Fontinettes, respectively.

The following subsections gather the modeling and control results for the system depicted in Fig. 3. However, for the sake of brevity, both the modeling and the control results are only presented for subsystem 1 (DDCR) in the paper. Results for subsystem 2 can be obtained in the same fashion as subsystem 1 .

\section{B. Modeling results}

The modeling approach is applied in two steps: in the first one, individual IDZ models are obtained for each single reach; in the second one, the interconnection formulas are applied to obtain the final models.

Equation (19) describes the model for the Douai-DonCuinchy reach. According to (20), the expressions for each sub-element of $\hat{P}(s)$ are computed. Again, due to lack of space, only $\hat{p}_{11}^{(G)}, \hat{p}_{12}^{(G)}$ and $\hat{p}_{13}^{(G)}$ are specified.

$$
\begin{aligned}
\hat{p}_{11}^{(G)}(s)= & \frac{-7.98 \cdot 10^{-4} s^{3}+4.617 \cdot 10^{-7} s^{2}}{s^{3}+8.426 \cdot 10^{-4} s^{2}+1.677 \cdot 10^{-7} s} \\
& +\frac{5.009 \cdot 10^{-10} s+7.6 \cdot 10^{-14}}{s^{3}+8.426 \cdot 10^{-4} s^{2}+1.677 \cdot 10^{-7} s} \\
\hat{p}_{12}^{(G)}(s)= & -\left(\frac{2.105 \cdot 10^{-3} s^{2}+2.018 \cdot 10^{-6} s}{s^{2}+5.203 \cdot 10^{-4} s}\right. \\
& \left.+\frac{2.675 \cdot 10^{-10}}{s^{2}+5.203 \cdot 10^{-4} s}\right) e^{-5.5 \cdot 10^{3} s} \\
\hat{p}_{13}^{(G)}(s)= & -\left(\frac{2.098 \cdot 10^{-3} s^{2}+1.129 \cdot 10^{-6} s}{s^{2}+3.223 \cdot 10^{-4} s}\right. \\
& \left.+\frac{1.265 \cdot 10^{-10}}{s^{2}+3.223 \cdot 10^{-4} s}\right) e^{-6.49 \cdot 10^{3} s}
\end{aligned}
$$

Note that the delays are rather large according to the length of the reaches.

\section{Control results}

According to Theorem 2, it is verified that this subsystem can be locally and globally stabilizable by a set of optimal output feedback controller defined in (6). After computation according to the expressions (24)-(26), the matrix relationship described in (8) is:

$$
K=\left[\begin{array}{ccc}
0.5118 & 0 & 0 \\
0 & -0.055 & 0 \\
0 & 0 & -0.077
\end{array}\right] \cdot 10^{3}
$$

The stabilizability proof of the system can be reached while verifying the conditions given in Theorem 2, such as

$$
\begin{gathered}
C r_{1}=[-0.64-0.0598-0.0177-0.034 \varepsilon \varepsilon \varepsilon \varepsilon \varepsilon]^{T} \\
C r_{2}=[-0.63-0.0597-0.0177-0.034-0.0009 \varepsilon \varepsilon \varepsilon \varepsilon]^{T}
\end{gathered}
$$


which satisfy (10) and (11), respectively, and $\varepsilon<-10^{-5}$ always. Thus, the local feedback action can suffice to stabilize each subsystem according to its desired performance.

Once the controller is designed, simulation results are provided for a realistic scenario in which there is a change of setpoint due to climate hazard such as a flood or a drought. This setpoint variation is applied to the whole system at the same time interval. Furthermore, additive noise is added (right part of Fig. 4) to see the global behavior of the decentralized controller according to an external disturbance and in order to prove the robustness of each local controller. Fig. 4 shows that the measured level matches the desired level with no significant error and in finite time.
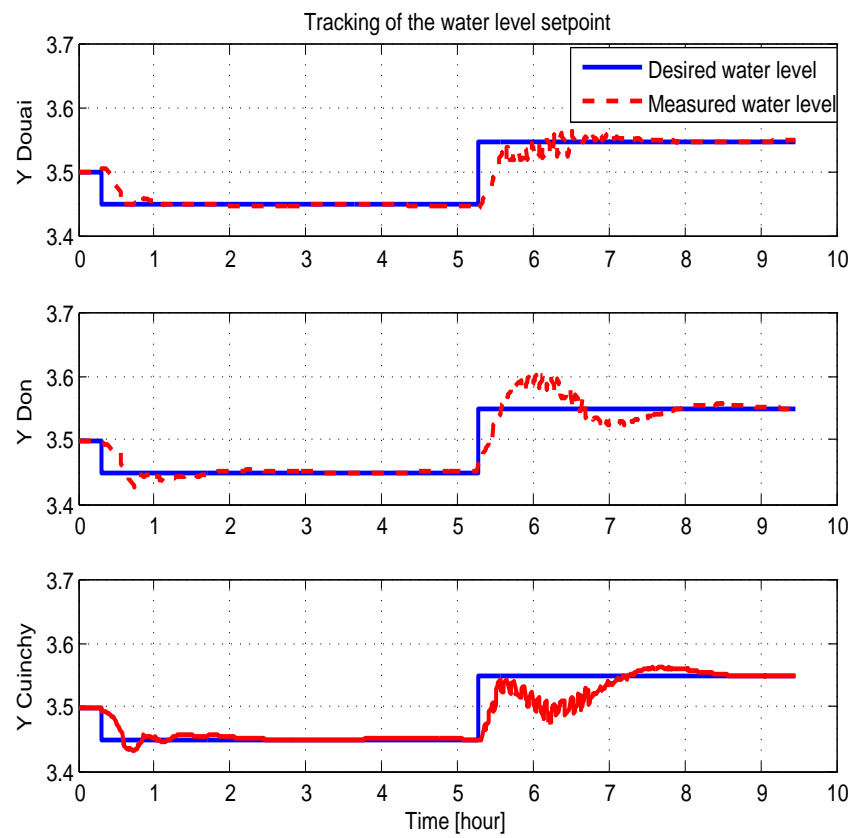

Fig. 4: Output dynamic with control action

To quantify the performance of the controller, consider the following tracking error indexes $T E$ given by:

$$
T E[\%]=100 *\left[1-\sqrt{\frac{1}{N} \sum_{i=1}^{N}\left(e_{i j}-\bar{e}_{i j}\right)^{2}}\right]
$$

where $N$ is the number of observed sample, $e_{i j}$ is the tracking error of the $j^{\text {th }}$ sample of the level $i$ and $\bar{e}_{i, j}$ is the mean of the same error. The tracking error is defined as the error between the setpoint and the measured level (blue and dashed red lines in Fig. 4, respectively).

The tracking performances for each water level setpoint are resumed in Table II. According to it, one can confirm that the control techniques provide successful results and the tracking performance of the system is guaranteed.

\begin{tabular}{c|ccc}
\hline \hline Water level & y Douai & y Don & y Cuinchy \\
\hline TE[\%] & 98.36 & 98.24 & 97.99 \\
\hline \hline
\end{tabular}

TABLE II: Tracking performance for each water level.

\section{CONCLUSION}

This work presented a decentralized control approach to ensure the efficient management of an inland navigation network. The objective consisted in maintaining the water level around a desired value close to the Normal Navigation Level (NNL). The control algorithm is developed for interconnected navigation reaches with distributaries, represented by Integral-Delay-Zero models, guaranteeing stability conditions. The proposed modeling procedure and control strategy are illustrated through their application in a navigation network located in the north of France.

\section{REFERENCES}

[1] D. Anda Ondo, L. Lefèvre and B. Chopard, Discrete Controllability of Distributed Parameters Systems using Lattice Boltzmann Models: an application to the Shallow Water Equations, IFAC World Congress, Milano, Italy, August 28 - September 2, 2011.

[2] Chow, V.T., Open-channel hydraulics. New York: McGraw-Hill, 1959.

[3] K. Horváth, E. Duviella, M. Petreczky, L. Rajaoarisoa and K. Chuquet, Model predictive control of water levels in a navigation canal affected by resonance waves, HIC 2014, New York, USA, August 17-21 2014.

[4] K. Horváth, E. Galvis, M. Valentín and J. Benedé, Is It Better to Use Gate Opening as Control Variable than Discharge to Control Irrigation Canals?, Journal of Irrigation and Drainage Engineering, March 2015, Vol. 141, no. 3.

[5] K. Horváth, M. Petreczky, L. Rajaoarisoa, E. Duviella and K. Chuquet, MPC of water level in a navigation canal - The Cuinchy-Fontinettes case study, ECC, Strasbourg, France, June 24-27, 2014.

[6] X. Litrico and V. Fromion, Simplified Modeling of Irrigation Canals for Controller Design, Journal of Irrigation and Drainage Engineering, pp 373-383, 2004.

[7] P.-O. Malaterre, Classification of canal control algorithms, Journal of Irrigation and Drainage Engineering, vol. 124, no. 1, pp. 310, 1998.

[8] P.-O. Malaterre, D. Dorchies and J.-P. Baume, Automatic tuning of robust PI controllers for a cascade of rivers or irrigation canals pools, ECC, Strasbourg, France, June 24-27, 2014.

[9] I. Mallidis, R. Dekker and D. Vlachos, The impact of greening on supply chain design and cost: a case for a developing region, Journal of Transport Geography, Volume 22, Pages 118-128, May 2012.

[10] I. Mareels, E. Weyer, S. Ooi, M. Cantoni, Y. Li, and G. Nair, Systems engineering for irrigation systems: Successes and challenges, Annual reviews in control, vol. 29, no. 2, pp. 191204, 2005.

[11] S. Mihic, M. Golusin and M. Mihajlovic, Policy and promotion of sustainable inland waterway transport in Europe - Danube River, Renewable and Sustainable Energy Reviews, Volume 15, Issue 4, Pages 1801-1809, May 2011.

[12] V. T. Pham, L. Lefèvre, D. Georges and G. Besançon (2014). Decentralized predictive control for $1 D$ cascaded systems of conservation laws, 19th IFAC WC, Cape Town, South Africa, August 24-29, 2014.

[13] V. Puig, J. Romera, J. Quevedo, C. M. Cardona, A. Salterain, E. Ayesa, I. Irizar, A. Castro, M. Lujan, P. Charbonnaud, P. Chiron and J.-L. Trouvat, Optimal predictive control of water transport systems: ArretDarre/Arros case study, Water science and technology, vol. 60, no. 8, pp. 21252133, 2009.

[14] C. Raievsky, V. T. Pham, J.-P. Jamont and L. Lefèvre, Irrigation canals distributed Model-based Predictive Control using multi-agent systems, MOSIM 2014, Nancy, France, November 2014.

[15] L. Rajaoarisoa, K. Horváth, E. Duviella and K. Chuquet, Large-scale system control based on decentralized design. Application to Cuinchy Fontinette Reach, IFAC WC, Cape Town, South Africa, August 24-29 2014.

[16] J. Rodellar, M. Gómez, and J. P. Martín Vide, Stable predictive control of open-channel flow, Journal of Irrigation and Drainage Engineering, vol. 115 , no. 4, pp. 701713, 1989.

[17] B. T. Wahlin, Performance of model predictive control on asce test canal 1, Journal of Irrigation and Drainage Engineering, vol. 130, no. 3, pp. 227238, 2004.

[18] S. Wright, Primal-Dual Interior-Point Methods. Society for Industrial and Applied Mathematics, 1997.

[19] MATLAB, version 7.10.0 (R2010a). Natick, Massachusetts: The MathWorks Inc., 2010.

[20] S. Boyd, L. El Ghaoui, E. Feron, and V. Balakrishnan. Linear Matrix Inequalities in System and Control Theory, SIAM, vol. 15, 1994. 